\title{
Real time PCR for the rapid identification and drug susceptibility of Mycobacteria present in Bronchial washings
}

Thilini Piushani Keerthirathne', Dhammika Nayoma Magana-Arachchi ${ }^{* *}$, Dushantha Madegedara² and Suneth Sithumini Sooriyapathirana ${ }^{3}$

\begin{abstract}
Background: Mycobacteria have a spectrum of virulence and different susceptibilities to antibiotics. Distinguishing mycobacterial species is vital as patients with non-tuberculous mycobacterial (NTM) infections present clinical features that are similar to those of patients with tuberculosis. Thus, rapid differentiation of Mycobacterium tuberculosis complex from NTM is critical to administer appropriate treatment. Hence the aim of the study was to rapid identification of mycobacterial species present in bronchial washings using multiplex real time Polymerase Chain Reaction (PCR) and to determine the drug susceptibility in identified mycobacterial species.
\end{abstract}

Methods: Sputum smear negative bronchoscopy specimens $(n=150)$ were collected for a period of one year, from patients attending the General Hospital Kandy, Sri Lanka. The specimens were processed with modified Petroff's method and were cultured on Löwenstein- Jensen medium. DNA, extracted from the mycobacterial isolates were subjected to a SYBR green mediated real time multiplex, PCR assay with primers specific for the M. tuberculosis complex, M. avium complex, M. chelonae-M.abscessus group and M. fortuitum group. DNA sequencing was performed for the species confirmation, by targeting the 16S rRNA gene and the drug susceptibility testing was performed for the molecularly identified isolates of M. tuberculosis and NTM.

Results: The optimized SYBR Green mediated multiplex real-time PCR assay was able to identify the presence of genus Mycobacterium in 25 out of 26 AFB positive isolates, two M. tuberculosis complex, three M. avium complex and two isolates belonging to $M$. chelonae-M. abscessus group. DNA sequencing confirmed the presence of $M$. tuberculosis, M. chelonae-M. abscessus, M. intracellulare, M. avium, Rhodococcus sp. and M. celatum. Remaining isolates were identified as Mycobacterium sp. All the NTM isolates were sensitive to amikacin and seven were resistant to ciproflaxacin. Twenty two of the NTM isolates and the isolate Rhodococcus was resistant to clarithromycin. The two isolates of $M$. tuberculosis were sensitive to all first line anti tuberculosis drugs.

Conclusion: The optimized SYBR Green mediated multiplex real time PCR assay could be an effective tool for the rapid differentiation of pathogenic M. tuberculosis complex from the opportunistic nontuberculous mycobacteria and also it confirmed the presence of NTM in $15.3 \%$ of the study population.

Keywords: Multiplex real-time PCR, MCAG, MAC, NTM, SYBR green

\footnotetext{
* Correspondence: cellbio@ifs.ac.lk

${ }^{1}$ National Institute of Fundamental Studies, Kandy, Sri Lanka

Full list of author information is available at the end of the article
} International License (http://creativecommons.org/licenses/by/4.0/), which permits unrestricted use, distribution, and reproduction in any medium, provided you give appropriate credit to the original author(s) and the source, provide a link to the Creative Commons license, and indicate if changes were made. The Creative Commons Public Domain Dedication waiver (http://creativecommons.org/publicdomain/zero/1.0/) applies to the data made available in this article, unless otherwise stated. 


\section{Background}

Tuberculosis (TB) is an infectious disease caused by strains of Mycobacterium tuberculosis complex (MTC) which is currently one of the leading causes of death in the world [1]. Alternatively, pulmonary infections caused by non-tuberculous mycobacteria (NTM), i.e. Mycobacterium species that are not members of MTC are also in the rise [2]. The increasing prevalence of the multi-drug resistant (MDR) TB strains are an added intimidation to the global TB burden, due to their resistance to the most effective first line anti-tuberculous drugs rifampin (RIF) and isoniazid (INH) [3]. The incidence of demises due to NTM related pulmonary infections have increased globally [4] and the rapid increase in the incidence of NTM disease in AIDS patients have also been recorded. Mycobacteria can be broadly classified as rapid growers ( $<7$ days) and slow growers ( $>7$ days) [5]. Fast growers commonly have two identical copies of the $16 \mathrm{~S}$ rRNA gene, whereas slow growers have only one single copy [6]. When a case of mycobacterial infection is detected, both the clinical and public health management depend critically on whether the causative agent is a MTC or a NTM to prevent clinical misleading during therapeutic actions, as patients with NTM infections show clinical signs that are similar to those of patients with TB [7]. Mycobacteria have a spectrum of virulence and different susceptibilities to antibiotics and it is believed that altered target proteins produced due to specific gene alterations such as mutations, insertions or deletions are influencing the degree of susceptibility to the drug [8]. Though it is anticipated that the patient's symptoms are mainly due to $M$. tuberculosis (MTB), the role of coinfecting NTM species in the pathogenesis of the pulmonary disease is still unclear. The presence of NTM in the patients with chronic TB has a substantial impact on clinical management. The treatment of diseases with MTC and NTM are different because many of the first and second line anti-tuberculous drugs are ineffective against many NTM [2]. Incorrect diagnosis of pulmonary diseases, i.e. if priority is given only for MTC and avoiding the presence of NTM, will lead to inappropriate treatments and the patients will not respond to conventional therapy [9].

As conventional detection methods such as, biochemical tests consume time [2], currently DNA amplification using Polymerase Chain Reaction (PCR) has allowed great progress in the rapid and accurate diagnosis of mycobacterial infections. Innovations in real -time PCR technology have eased and advanced the PCR methodology extensively. Application of SYBR green mediated real time PCR assay in clinical microbiology had improved the diagnostics due to the increased specificity and the ability to detect two or more organisms in a single reaction [10]. Rapid availability of the results is an added advantage of SYBR green mediated real time PCR assay over conventional PCR. Rapid identification will assist in avoiding unnecessary drug exposure and could aid in reducing the mortality and morbidity.

Incidence of TB in Sri Lanka was 66 in 2013. Human immunodeficiency virus (HIV) testing of all TB patients was made mandatory in the country since 2013. In 2013, 4646 TB patients were screened for HIV and out of these patients, six were found positive. In addition, there were nine patients with known HIV status at the time of diagnosis of $\mathrm{TB}$, contributing to a total of 15 patients with HIV/ TB co-infection in 2013 [11]. In Sri Lanka, highest priority is given for the definitive diagnosis of TB and due to the limited records [12] about the NTM in clinical specimens, no testing is carried out for definitive diagnosis of NTM infections. Therefore any person with symptoms suggestive of $\mathrm{TB}$, particularly cough for more than three weeks are being investigated only for TB and similar to other developing countries, routine diagnosis of TB in Sri Lanka is done with the sputum microscopy which could lead to the misdiagnosis of the pulmonary disease.

In our previous study we demonstrated the usefulness of bronchial washings as the preferred specimen in diagnosis of NTM lung disease by culture as well as from molecular techniques and about 13 to $14 \%$ of the study population had NTM in their bronchial washings [12]. Hence in this study we assessed the utility of SYBR green mediated multiplex, real time PCR for the rapid identification of Mycobacterium species present in bronchial washings and also determined the drug sensitivity patterns of the identified mycobacterial isolates.

\section{Methods}

\section{Study setting, population and ethics}

Sputum smear negative bronchoscopy specimens were collected from patients $(n=150)$ attending the General Hospital Kandy, Sri Lanka from January 2014 to March 2015 who had pulmonary symptoms, nodular or cavitary opacities on chest radiograph, or an HRCT scan that showed multifocal bronchiectasis with multiple small nodules. Approval for the study was obtained from the Ethical Review Committee of the Postgraduate Institute of Science, University of Peradeniya, Sri Lanka. The bronchoalvelar lavage (BAL) specimens were collected as a part of routine investigations and a portion of the specimens were used for culturing. Written informed consent was obtained from each patient before bronchoscope and collecting the BAL specimens.

\section{Laboratory analysis}

Sample processing, culture and DNA extraction

The samples were liquefied and decontaminated with $4 \% \mathrm{NaOH}$ according to the modified Petroff's method 
[13]. The decontaminated specimens were inoculated onto Löwenstein-Jensen media (L-J media), LJ media containing Thiophene - 2 carboxylic acid hydrazide $(\mathrm{TCH})$ and p-nitrobenzioc acid (PNB) and cultures were observed for 8 to 12 weeks incubating at $37{ }^{\circ} \mathrm{C}$ and at $28{ }^{\circ} \mathrm{C}$ in light and dark conditions. Solid medium slants were considered positive when visible colonies grew. The colonies were further confirmed as mycobacteria by the Ziehl-Neelsen stain. All cultures that showed mycobacterial growth were subjected to further analysis. Genomic DNA was extracted from the mycobacterial isolates according to the standard CTAB (N-Cetyl-N, $\mathrm{N}, \mathrm{N}$-trimethyl ammonium bromide) method [14].

\section{Real - time multiplex PCR analysis}

PCR primers targeting the internal transcribed spacers (ITSs) of MTC and $M$. chelonae-M. abscessus group (MCAG), the 16S rRNA genes of $M$. avium complex (MAC), M. fortuitum group (MFG) and the region of the 16SrRNA gene common to all the members of genus Mycobacterium which were described previously [10] were used for the optimizing of real -time multiplex, PCR assays (Table 1). The assays were performed on a real time PCR Instrument System (Rotor-GeneQ) and the DNA amplifications were monitored by the measurement of the SYBR Green fluorescence. The real time multiplex PCR assay was conducted in two separate reactions where primers specific for MTC and MAC were in reaction I and primers specific for MCAG and MFG were in reaction II which helped in identifying slow and rapid growers respectively. Primers targeting genus

Table 1 Primers used for SYBR Green mediated, multiplex realtime PCR assay [10]

\begin{tabular}{|c|c|c|c|}
\hline Organism & $\begin{array}{l}\text { Target } \\
\text { region }\end{array}$ & Primer sequence $\left(5^{\prime}-3^{\prime}\right)$ & Reaction \\
\hline \multirow{2}{*}{$\begin{array}{l}\text { Mycobacterium } \\
\text { avium }\end{array}$} & \multirow[t]{2}{*}{$16 S$} & F:CCTCAAGACGCATGTCTTC & \multirow[t]{2}{*}{ I } \\
\hline & & R: ACCTACCGTCAATCCGAGAA & \\
\hline \multirow{2}{*}{$\begin{array}{l}\text { Mycobacterium } \\
\text { intracelulare }\end{array}$} & \multirow[t]{2}{*}{$16 S$} & F:GACCTITAGRCGCATGTCTIT & \multirow[t]{2}{*}{1} \\
\hline & & R: ACCTACCGTCAATCCGAGAA & \\
\hline \multirow{2}{*}{$\begin{array}{l}\text { Mycobacterium } \\
\text { tuberculosis } \\
\text { Complex }\end{array}$} & \multirow[t]{2}{*}{ ITS } & F: GCGAGAGCCGGGTGCATG & \multirow[t]{2}{*}{ I } \\
\hline & & R: AACAGTGTGTTGGTGGCCAA & \\
\hline \multirow[t]{2}{*}{ AFB genus } & \multirow[t]{2}{*}{$16 S$} & F: CCGCAAGRCTAAAACTCAAA & \multirow[t]{2}{*}{$|/| \mid$} \\
\hline & & $\begin{array}{l}\text { R: } \\
\text { TGCACACAGGCCACAAGGGA }\end{array}$ & \\
\hline \multirow[t]{2}{*}{ MCAG } & \multirow[t]{2}{*}{ ITS } & F: TAAGGAGCACCATITCCCAG & \multirow[t]{2}{*}{$\|$} \\
\hline & & R: CGACGTIITGCCGACTACC & \\
\hline \multirow[t]{4}{*}{ MFG } & \multirow[t]{4}{*}{$16 S$} & F: CCACGCGCTTCATGGTGT & \multirow[t]{4}{*}{$\|$} \\
\hline & & F: CCGCGCTCTTCATGGGGT & \\
\hline & & F: ACCACGCATTTCATGGTGT & \\
\hline & & R:ACTTGCGCTTCGTCCCTAT & \\
\hline
\end{tabular}

Mycobacterium were included in both the reactions (Table 1). Each reaction was carried out in a $25 \mu \mathrm{l}$ volume which contained $2.0 \mu \mathrm{l}$ of $25 \mathrm{mM} \mathrm{MgCl}_{2}, 0.25 \mu \mathrm{l}$ of $5 \mathrm{u} / \mu \mathrm{l}$ Taq polymerase, $5.0 \mu \mathrm{l}$ of $5 \mathrm{X}$ PCR Buffer, $2.5 \mu \mathrm{l}$ of $1 \mathrm{mM}$ dNTP mix, $1.0 \mu \mathrm{l}$ of each primer $(10 \mu \mathrm{m}), 1.25 \mu \mathrm{l}$ of $2 \mathrm{X}$ SYBR green and $50 \mathrm{ng}$ of the extracted mycobacterial DNA. The PCR amplification process was initiated by ramping the temperature at $95{ }^{\circ} \mathrm{C}$ for $5 \mathrm{~min}$ followed by 40 cycles of the amplification process $\left(95{ }^{\circ} \mathrm{C}\right.$ for $15 \mathrm{~s}$, $60{ }^{\circ} \mathrm{C}$ for 30 s and $72{ }^{\circ} \mathrm{C}$ for 30 s). Subsequent to the cycling process, melting curves were generated by inclining the temperature from $60{ }^{\circ} \mathrm{C}$ to $95{ }^{\circ} \mathrm{C}$ at $0.2^{\circ} \mathrm{C} / \mathrm{s}$.

\section{DNA sequencing for species confirmation}

For the species confirmation, the extracted DNA of the mycobacterial isolates were subjected to amplification of the 16S rRNA gene with the use of universal primers, $\mathrm{F}$ : 5' TGGAGAGTTTGATCCTGGCTCAG 3' and R: 5' AAGGAGGTGATCCATC 3' [10]. The amplified DNA was visualized from gel documentation system (SYNGENE) after the electrophoresis in $1.5 \%$ agarose and ethidium bromide staining. $1 \mathrm{kbp}$ DNA marker was used to identify correct fragment of $1.5 \mathrm{kbp}$. Amplified DNA fragments were purified using gel extraction kit (Promega) and were commercially sequenced from Macrogen Inc., South Korea using ABI 3730XL sequencers. The sequence data obtained were analysed using the software program BioEdit 7.0.9. The results were compared with the sequences from the GenBank database and the obtained sequences were deposited in the GeneBank database.

\section{Drug susceptibility analysis}

The drug susceptibility testing for both MTC $(n=2)$ and NTM $(n=22)$ were carried out in duplicate with freshly grown cultures using the methods of agar proportion and the disk diffusion [15]. Susceptibility to isoniazid (INH) $(0.2 \mu \mathrm{g} / \mathrm{ml}, 1.0 \mu \mathrm{g} / \mathrm{ml})$, rifampin (RIF) $(0.2 \mu \mathrm{g} / \mathrm{ml})$, streptomycin $(2 \mu \mathrm{g} / \mathrm{ml}, 10 \mu \mathrm{g} / \mathrm{ml})$, ethambutol $(5.0 \mu \mathrm{g} /$ $\mathrm{ml}, 10 \mu \mathrm{g} / \mathrm{ml})$ and pyrazinamide $(25 \mu \mathrm{g} / \mathrm{ml})$ were determined with the agar proportion method [16] performed on Middle Brook 7H11 (MB7H11) agar media enriched with the oleic albumin dextrose catalase (OADC) supplement. The standard strain of $\mathrm{H}_{37} \mathrm{Rv}$ was used as the positive control. MB7H11 agar plates of 4-5 mm thickness, enriched with the OADC supplement was used for the susceptibility testing of the drugs amikacin $(30 \mu \mathrm{g})$, ciprofloxacin $(5 \mu \mathrm{g})$ and clarithromycin $(15 \mu \mathrm{g})$ incorporated disks, purchased commercially from Hardy Diagnostics, Santa Maria, CA. At the same time, the viability of the disks were tested using the control strains of Escherichia coli ATCC 25922, Pseudomonas aeruginosa ATCC 27853 and Staphylococcus aureus ATCC 25923 on MuellerHinton Agar medium. Susceptibility patterns of these organisms were compared as stated in Clinical and 
Laboratory Standards Institute standard operating procedures [14].

\section{Results}

Participants and the culture characteristics

Of the 150 patients included in the study, 93 were males. The men: women ratio was 1.6:1. The age range was 12 to 83 years. $32 \%$ of the males were above the age of 60 years while in females only $11 \%$ were above the age of 60 years. But in the age group of 15-60 years, female percentage was higher $(32 \%)$ in comparison to the male patients $(24 \%)$. Of the 150 specimens, 52 had a growth within eight weeks of incubation. Overall, twenty three of 150 patients (15.3\%) were positive for any Mycobacterium species by culture (by confirmation of AFB). Out of these 23 patients, 14 were females $(60.8 \%)$ and MTB was identified in two of them. When mycobacterial culture was considered as the gold standard there were 26 isolates (17.3 \%) from 23 patients belonging to the Mycobacterium genus and among theses 23 (15.3\%) were NTM. All the mycobacterial cultures were rapid growers except for two isolates. Mixed growths were observed from the two bronchial wash specimens. The sample $\mathrm{KCC} 2$ yielded three different isolates (02RC, 02YC and 02WC) and the sample KCC 6 yielded two isolates (06 RC and 06PL) and all these culture isolates were confirmed as AFB positive by $\mathrm{ZN}$ staining.

\section{Analysis of SYBR green mediated real time multiplex PCR assay}

Initial assessment of the PCR primers for the specificity and the sensitivity of the genus Mycobacterium and MTC was done in a single-plex real time PCR with a standard $\mathrm{H} 37 \mathrm{Rv}$ strain, an isolate of $M$. intracellulare and M. phocaicum, and a collection of previously identified clinical isolates of MTB. The presence of the amplicon was detected by the fluorescent dye SYBR green and the species specificities were defined by the analysis of melting curves. In Reaction 1, a DNA template from MTB produced a peak with a $T_{m} \sim 77{ }^{\circ} \mathrm{C}$, which corresponded to MTC, and a peak with a $T_{m} \sim 80-83{ }^{\circ} \mathrm{C}$ which corresponded to the genus Mycobacterium respectively. Similarly DNA from MAC produced a peak with a $T_{m} \sim 85^{\circ} \mathrm{C}$, which corresponded to MAC (Fig. 1a). In Reaction II, a DNA template from MCAG produced a peak with a $T_{m} \sim$ $77^{\circ} \mathrm{C}$, which corresponded to MCAG, and a peak with a $T_{m} \sim 80-83{ }^{\circ} \mathrm{C}$ corresponded to the genus Mycobacterium respectively. Similarly in Reaction II, DNA from Mycobacterium spp. produced peaks with $T_{m} \sim 84-89{ }^{\circ} \mathrm{C}$, which corresponded to MFG and other mycobacteria and a peak with a $T_{m} \sim 80-83{ }^{\circ} \mathrm{C}$ which corresponded to the genus Mycobacterium (Fig. 1b). When the mycobacterial DNA of the study population were subjected to Reaction I of the SYBR green mediated real time multiplex PCR assay, it confirmed the presence of Mycobacterium genus in 25 of the 26 AFB positive isolates, in two MTC $(n=2)$ isolates and three MAC $(n=3)$ isolates. Evaluation of the analysis of Reaction II also confirmed the presence of genus Mycobacterium in tested mycobacterial isolates and also the presence of MCAG in two isolates $(n=2)$.

\section{Analysis of DNA sequencing}

DNA sequencing confirmed the presence of a Rhodococcus sp. $(n=1)$, a Mycobacterium celatum $(n=1)$, M. chelonae-abscessus complex $(n=3)$, two isolates were identified as $M$. intracellulare and the other isolate as $M$. avium. The isolates identified as MTC were confirmed as MTB and the remaining isolates could be only identified as belonging to genus Mycobacterium.

\section{Analysis of drug susceptibility testing}

All the NTM isolates were sensitive to amikacin and seven were resistant to ciproflaxacin. Except for the two isolates of $M$. chelonae-abscessus complex and a single isolate of M. intracellulare, all the other isolates of NTM were resistant to clarithromycin (Table 2). None of the isolates were resistant to all three tested drugs. The two isolates of $M$. tuberculosis were sensitive to all first line anti tuberculosis drugs.

\section{Discussion}

Conclusive diagnosis of a mycobacterial infection commonly involves isolation and identification of the infecting organism in the laboratory by culture, even though, the preliminary diagnosis is frequently based on clinical data [17]. But even when cultures are detected positive and the presence of mycobacteria were confirmed by $\mathrm{ZN}$ staining it is not yet possible to confirm it as a MTC or a NTM [18]. To address this issue we have optimized a molecular assay capable of identifying MTC and NTM when applied to positive cultures on LJ; further identification of species is achieved by the sequencing of 16SrDNA sequencing. In this study, 25 clinical isolates were identified in terms of species, group or to complex level with the SYBR green mediated real time multiplex, PCR assay, keeping $16 \mathrm{~S}$ rRNA gene sequencing as the gold standard. The PCR assay indicated the presence of two MTB isolates and 23 NTM isolates in the collected bronchial washings. However $16 \mathrm{~S}$ rRNA gene sequencing confirmed that one of the isolate was belonging to the genus Rhodococcus which was positive in $\mathrm{ZN}$ stain. $16 \mathrm{~S}$ amplicons from two NTM positive cultures failed to yield DNA sequence data and as such further identification of these two isolates could not be made. When mycobacterial culture was considered as the gold standard there were 26 isolates (17.3\%) belonging to the Mycobacterium genus and among theses 23 (15.3\%) were NTM. 

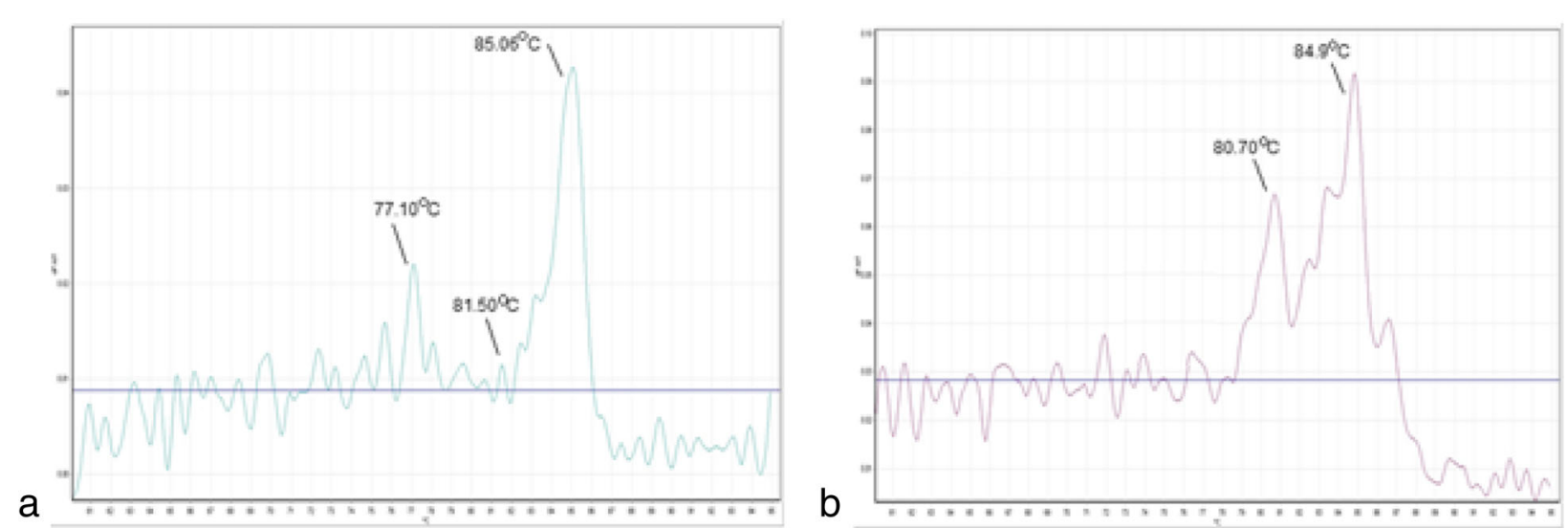

Fig. 1 a SYBR green mediated real-time PCR melting curve, $\operatorname{Tm} 77.10^{\circ} \mathrm{C}, 81.50^{\circ} \mathrm{C}$ and $85.06^{\circ} \mathrm{C}$ indicating the presence of MTC, genus Mycobacterium and MAC following Reaction I. b SYBR green mediated real-time PCR melting curve, $\mathrm{Tm} 80.70{ }^{\circ} \mathrm{C}$ and $84.90^{\circ} \mathrm{C}$ indicating the presence of genus Mycobacterium and MFG following Reaction II

In the past two decades, nucleic acid amplificationbased techniques such as PCR have become accessible to the clinical mycobacteriology laboratory. Sequence based methods are more rapid and accurate than the conventional identification methods. Most PCR protocols have concentrated on the detection of pathogenic MTB as they claim more human lives than any other bacteria. Both in house and commercial PCRs have been widely evaluated $[1,2,10,19]$. Real-time PCR techniques, involving fluorescent dyes or fluorophores with a spectrofluorometric

Table 2 Effect of clarithromycin, amikacin and ciprofloxacin on the identified NTM isolates

\begin{tabular}{|c|c|c|c|c|c|}
\hline \multirow[t]{2}{*}{ SI number } & \multirow[t]{2}{*}{ Isolate } & \multirow[t]{2}{*}{ Organism } & \multicolumn{3}{|l|}{ Drug } \\
\hline & & & Clarithromycin & Amikacin & Ciprofloxacin \\
\hline 1. & KCC 142 & Mycobacterium sp. (KU198858) & $\mathrm{R}$ & $\mathrm{S}$ & $\mathrm{S}$ \\
\hline 2. & KCC 06 PL & Mycobacterium chelonae-abscessus complex (KU179046) & S & S & $\mathrm{R}$ \\
\hline 3. & KCC 124 & Mycobacterium chelonae-abscessus complex (KU195328) & S & S & $\mathrm{R}$ \\
\hline 4. & KCC 06 RC & Mycobacterium chelonae-abscessus complex (KU195330) & $\mathrm{R}$ & S & $\mathrm{R}$ \\
\hline 5. & KCC119 & Mycobacterium celatum (KU179047) & S & S & $\mathrm{R}$ \\
\hline 6. & KCC 02 YC & Rhodococcus sp. (KU198859) & $\mathrm{R}$ & S & $\mathrm{S}$ \\
\hline 7. & KCC 72 & Mycobacterium sp.(KU195326) & $\mathrm{R}$ & S & $\mathrm{R}$ \\
\hline 8. & KCC 82 & Mycobacterium sp.(KU195322) & $\mathrm{R}$ & S & $S$ \\
\hline 9. & KCC 131 & Mycobacterium avium complex(KU198858) & $\mathrm{R}$ & S & S \\
\hline 10. & KCC 94 & Mycobacterium sp.(KU377301) & $\mathrm{R}$ & S & S \\
\hline 11. & KCC 98 & Mycobacterium sp.(KU195324) & $\mathrm{R}$ & S & S \\
\hline 12. & KCC 90 & Mycobacterium intracellulare (KU195323) & S & S & S \\
\hline 13. & KCC 101 & Mycobacterium sp.(KU195327) & $\mathrm{R}$ & S & S \\
\hline 14. & KCC 78 & Mycobacterium sp.(KU195320) & $\mathrm{R}$ & S & $\mathrm{R}$ \\
\hline 15. & KCC 83 & Mycobacterium sp. (KU377300) & R & S & S \\
\hline 16. & KCC 129 & Mycobacterium sp.(KU195325) & $\mathrm{R}$ & S & S \\
\hline 17. & KCC 76 & Mycobacterium intracellulare (KU195319) & R & $S$ & $S$ \\
\hline 18. & KCC 81 & Mycobacterium sp.(KU195321) & $\mathrm{R}$ & S & S \\
\hline 19. & KCC 97 & Mycobacterium sp. (KU377299) & R & $S$ & $S$ \\
\hline 20. & KCC 59 & Mycobacterium sp. (KU195318) & $\mathrm{R}$ & S & S \\
\hline 21. & KCC 132 & Mycobacterium sp. & $\mathrm{R}$ & S & S \\
\hline 22. & KCC 73 & Mycobacterium sp. & $\mathrm{R}$ & S & $\mathrm{R}$ \\
\hline
\end{tabular}


thermal cycler, have been used to develop a number of rapid and sensitive assays for identification of bacteria and viruses [20]. Several fluorescence formats are available for the detection of amplified DNA of MTB. Most of these assays have used SYBR Green, a double-stranded DNA (dsDNA) binding dye that fluoresces when bound to dsDNA [21]. Fluorimeter-based analysis also has provided a rapid and sensitive method for identification of PCR products [21]. However, even species specific realtime PCR, have their limitations since it only recognises selected species of interest.

The molecular identification procedure which was elucidated in this study intended to identify rapid and slow growing mycobacteria in two separate reactions. Hence this assay was able to differentiate the pathogenic mycobacterial presence with the use of these two parallel reactions. Including the Mycobacterium genus specific primer set in both reactions was to use it as a positive internal control which facilitated the identification of the presence of various Mycobacterium species, though the absence of the targeted organisms. A third of the world population is thought to be infected with TB [21]. The developing countries including Sri Lanka, are still suffering from TB, mainly due to poverty (lack of healthy living conditions) and satisfactory medical care [22]. There were two $M$. tuberculosis strains identified during the study, both the strains were sensitive for the first line anti TB drugs indicating that those were treatable tuberculosis cases. DNA from the identified M. tuberculosis isolates were amplified, sequenced and deposited on to the GenBank data base with the accession numbers KU179045 and KU195329.

In this study a considerable proportion of the isolates were identified as NTM. These were identified as slender pink rods upon $\mathrm{ZN}$ staining which is still used as the preliminary diagnostic tool in most of the developing countries mainly due to the cost effectiveness and easy performance [23]. The mycobacterial infections due to these organisms might present similar clinical symptoms mimicking $\mathrm{TB}$, making the infection misdiagnosed as $\mathrm{TB}$, hence therapy would include anti-tuberculous drugs. Precise identification of the Mycobacterium species before treatment can have a considerable influence on clinical management. Misdiagnosis of NTM bacilli as MTB and thereby considering it as pulmonary TB, can lead to unnecessary treatment of patients which could lead to the emergence of drug resistant Mycobacterium strains. Moreover, Rhodococcus spp which was identified is also an acid fast bacterium [24] which renders it difficult to discriminate from MTB and NTM. Therefore molecular identification methods are beneficial in the identification of such species prior to initiation of treatment.

There are incidences where $M$. celatum isolates had been isolated from bronchial washings [25] which was elucidated as a potential human pathogen [26]. M. celatum was first reported in 1993. This bacterium causes pulmonary infections similar to the infections caused by MTB and other NTM [19]. Even though, it was believed that this organism causes infection in patients who have depressed immunity there were reports describing pulmonary infections caused by $M$. celatum in immuno-competent patients [27]. The isolate from this study produced smooth, convex, non-pigmented colonies on L-J medium. Even though this species is known as a slow grower, the $M$. celatum isolate identified (KU179047) during this study was found to be rapidly growing bacterium which formed colonies on L-J medium in less than 7 days. The isolate was resistant to ciprofloxacin.

M. avium, $M$. intracellulare, $M$. chelonae, $M$. abscessus and $M$. fortuitum are the most common NTM pathogens found to be causing pulmonary infections [4]. Infections caused by MAC (M. avium and $M$. intracellulare) could lead to respiratory failure if untreated [28]. Increase in incidence of MAC was reported in early 1980's simultaneously with the beginning of the AIDS widespread [29]. Though the two species, $M$. avium and $M$. intracellulare belong to a same group, in their pathogenicity and biology they show significant differences [30]. During this study three isolates were identified as MAC by multiplex Realtime PCR assay, but when sequenced, two isolates were confirmed as $M$. intracellulare and the other as M. avium. The $M$. avium isolate (KU198857) and one of the $M$. intracellulare isolates (KU195319) were resistant to the drug clarithromycin while the other M.intracellulare isolate (KU195323) was sensitive to all the tested drugs.

Infections caused by MCAG consisting of rapidly growing mycobacteria are a serious public health problem. MCAG consists of $M$. immunogenum, $M$. massiliense, M. bolletii, and M. salmoniphilum apart from $M$. abscessus and M. chelonae [10]. These species cannot be differentiated by biochemical testing and their $16 \mathrm{~S}$ rRNA gene sequences are often similar, which renders it difficult to discriminate them individually. There were three MCAG isolates (KU179046, KU195328, and KU195330) identified during this study in which two isolates were resistant to ciprofloxacin while the other was resistant to both the clarithromycin and ciprofloxacin.

In most of the developing countries AFB positive smear microscopy is the tool to initiate anti TB treatment. However before the availability of the culture reports patients clinical conditions may deteriorate due to many factors such as side effects of anti TB therapy, drug resistance or if suffering from an infection due to NTM. Thus the results of this described molecular assay can help to expedite the decision making process so that relevant treatment could be initiated without delay. 


\section{Conclusion}

In conclusion, the optimized SYBR green mediated multiplex real time PCR procedure described, is rapid and simple to perform and could assist in identifying and differentiation of pathogenic $M$. tuberculosis complex from the opportunistic non tuberculous mycobacteria, which will allow early initiation of treatment depending on the causative agent.

\section{Abbreviations}

AFB: Acid fast bacilli; CTAB: N-Cetyl-N, N, N-trimethyl ammonium bromide; INH: Isoniazid; ITs: Internal transcribed spacers; L-J medium: LöwensteinJensen medium; MAC: M. avium complex; MCAG: M. chelonae-M.abscessus group; MDR: Multi-drug resistant; MFG: M. fortuitum group; MTC: Mycobacterium tuberculosis complex; NTM: Non-tuberculous Mycobacteria; PCR: Polymerase chain reaction; PNB: P-nitrobenzioc acid RIF: Rifampin; TB: Tuberculosis; TCH: Thiophene -2 carboxylic acid hydrazide; ZN stain: Ziehl-Neelsen stain

\section{Acknowledgements}

The authors gratefully acknowledge the National Research Council, Sri Lanka, grant number 11-059 for providing the equipment Real-Time PCR and the Department of Microbiology, Faculty of Medicine, University of Peradeniya, Sri Lanka for providing the standard microbial strains necessary for the Disk diffusing method.

\section{Funding}

This study was funded by the National Institute of Fundamental Studies, Sri Lanka.

\section{Availability of data and materials}

The datasets during and/or analysed during the current study available from the corresponding author on reasonable request. The datasets generated during and/or analysed during the current study are available in the [GenBank] repository for the accession numbers listed in the text.

\section{Authors' contributions}

TK carried out the molecular techniques, drug susceptibility testing and drafted the manuscript. DNM concept, designed and supervised the study, coordinated, corrected and revised the manuscript after the reviewers' comments. DM screened and recruited the patients and assisted in the sample collection. SS made corrections to the manuscript. All authors read and approved the final manuscript.

\section{Competing interests}

The authors declare that they have no competing interests.

\section{Consent for publication}

Not applicable.

\section{Ethics approval and consent to participate}

Approval for the study was obtained from the Ethical Review Committee of the Postgraduate Institute of Science, University of Peradeniya, Sri Lanka. The bronchoalvelar lavage (BAL) specimens were collected as a part of routine investigations and a portion of the specimens were used for culturing. Written informed consent was obtained from each patient before bronchoscope and collecting the BAL specimens.

\section{Author details}

${ }^{1}$ National Institute of Fundamental Studies, Kandy, Sri Lanka. ${ }^{2}$ Respiratory Disease Treatment Unit \&Teaching Hospital, Kandy, Sri Lanka. ${ }^{3}$ Department of Molecular Biology and Biotechnology, University of Peradeniya, Peradeniya, Sri Lanka.

Received: 7 January 2016 Accepted: 19 October 2016 Published online: 26 October 2016

\section{References}

1. Moon SH, Kim EJ, Tomono J, Miyamoto S, Mitarai S, Kim DW, Seki M. Detection of Mycobacterium tuberculosis complex in sputum specimens using a loop-mediated isothermal amplification assay in Korea. J Med Microbiol. 2015;64:1335-40.

2. Gupta S, Bandyopadhyay D, Paine SK, Gupta S, Banerjee S, Bhattacharya S, Gachhui R, Bhattacharya B. Rapid identification of mycobacterium species with the aid of multiplex polymerase chain reaction (PCR) from clinical isolates. Open Microbiol J. 2010;4:93-7.

3. Mulisa G, Workneh T, Hordofa N, Suaudi M, Abebe G, Jarso G. Multidrugresistant Mycobacterium tuberculosis and associated risk factors in Oromia Region of Ethiopia. Int J Infect Dis. 2015;39:57-61.

4. $\quad Y u X L$, Lu L, Chen GZ, Liu ZG, Lei H, Song YZ, Zhang SL. Identification and characterization of non-tuberculous mycobacteria isolated from tuberculosis suspects in Southern-central China. PLoS One. 2014;9:e114353.

5. García-Agudo L, García-Martos P. Clinical significance and antimicrobial susceptibility of rapidly growing mycobacteria. Sci Against Microb Pathog Commun Curr Res Technol Adv. 2011; 363-77.

6. Reischl U, Feldmann K, Naumann L, Gaugler BJ, Ninet B, Hirschel B, Emler S. $16 \mathrm{~S}$ rRNA sequence diversity in Mycobacterium celatum strains caused by presence of two different copies of 165 rRNA gene. J Clin Microbiol. 1998;36:1761-4

7. Hong YJ, Chung YH, Kim TS, Song SH, Park KU, Yim JJ, Song J, Lee JH, Kim EC. Usefulness of three-channel multiplex real-time PCR and melting curve analysis for simultaneous detection and identification of the Mycobacterium tuberculosis complex and nontuberculous mycobacteria. J Clin Microbiol. 2011;49:3963-6.

8. Louw GE, Warren RM, van Pittius NC G, McEvoy CR, Van Helden PD, Victor TC. A balancing act: efflux/influx in mycobacterial drug resistance. Antimicrob Agents Chemother. 2009;53:3181-9.

9. Maiga M, Siddiqui S, Diallo S, Diarra B, Traore B, Shea YR, Zelazny AM, Dembele BP, Goita D, Kassambara H, et al. Failure to recognize nontuberculous mycobacteria leads to misdiagnosis of chronic pulmonary tuberculosis. PLoS One. 2012:7:e36902.

10. Richardson ET, Samson D, Banaei N. Rapid Identification of Mycobacterium tuberculosis and nontuberculous mycobacteria by multiplex, real-time PCR. J Clin Microbiol. 2009;47:1497-502.

11. Annual Report of the National Programme for Tuberculosis Control and Chest Diseases. Sri Lanka: Ministry of Health, 2013. Available from: http:// www.nptccd.health.gov.|k/uploaded/documents/ Annual\%20Report\%20of\%20NPTCCD\%202013.pdf. Accessed 30 May 2016.

12. Weerasekera DK, Magana-Arachchi DN, Madegedara D, Dissanayake N. Polymerase chain reaction - restriction fragment length polymorphism analysis for the differentiation of mycobacterial species in bronchial washings. Ceylon Med J. 2014;59:79-83.

13. Tripathi K, Tripathi PC, Nema S, Shrivastava AK, Dwiwedi K, Dhanvijay AK Modified Petroff's Method: an Excellent Simplified Decontamination Technique in Comparison with Petroff's Method. Int J Recent Trends Sci Tech. 2014;10:461-4.

14. Somerville W, Thibert L, Schwartzman K, Behr MA. Extraction of Mycobacterium tuberculosis DNA: a question of containment. J Clin Microbiol. 2005:43:2996-7.

15. Wallace Jr RJ, Dalovisio JR, Pankey GA. Disk diffusion testing of susceptibility of Mycobacterium fortuitum and Mycobacterium chelonei to antibacterial agents. Antimicrob Agents Chemother. 1979;16:611-4.

16. Nici L, Donner C, Wouters E, Zuwallack R, Ambrosino N, Bourbeau J, Carone M, Celli B, Engelen M, Fahy B. American thoracic society/European respiratory society statement on pulmonary rehabilitation. Am J Resp Crit Care Med. 2006;173:1390-413

17. Soini H, Musser JM. Molecular diagnosis of mycobacteria. Clin Chem. 2001;47:809-14.

18. Cheah ES, Malkin J, Free RC, Lee SM, Perera N, Woltmann G, Patel H, Kimmitt PT, Smith RJ, Rajakumar K, et al. A two-tube combined TaqMan/SYBR Green assay to identify mycobacteria and detect single global lineage-defining polymorphisms in Mycobacterium tuberculosis. J Mol Diagn. 2010;12:250-6.

19. Piersimoni C, Zitti PG, Nista D, Bornigia S. Mycobacterium celatum pulmonary infection in the immunocompetent: case report and review. Emerg Infect Dis. 2003;9:399-402.

20. Miller N, Cleary T, Kraus G, Young AK, Spruill G, Hnatyszyn HJ. Rapid and specific detection of Mycobacterium tuberculosis from acid-fast bacillus smear-positive respiratory specimens and BacT/ALERT MP culture bottles by using fluorogenic probes and real-time PCR. J Clin Microbiol. 2002;40:4143-7. 
21. Lachnik J, Ackermann B, Bohrssen A, Maass S, Diephaus C, Puncken A, Stermann M, Bange FC. Rapid-cycle PCR and fluorimetry for detection of mycobacteria. J Clin Microbiol. 2002;40:3364-73.

22. Smith I. Mycobacterium tuberculosis pathogenesis and molecular determinants of virulence. Clin Microbiol Rev. 2003;16:463-96.

23. Patiño S, Alamo L, Cimino M, Casart Y, Bartoli F, García MJ, Salazar L. Autofluorescence of mycobacteria as a tool for detection of Mycobacterium tuberculosis. J Clin Microbiol. 2008;46:3296-302.

24. Spiliopoulou A, Assimakopoulos SF, Foka A, Kolonitsiou F, Lagadinou M, Petinaki E, Anastassiou ED, Spiliopoulou I, Marangos M. Pulmonary infection by Rhodococcus equi presenting with positive Ziehl-Neelsen stain in a patient with human immunodeficiency virus: a case report. J Med Case Rep. 2014;8:423.

25. Couto I, Machado D, Viveiros M, Rodrigues L, Amaral L. Identification of nontuberculous mycobacteria in clinical samples using molecular methods: a 3-year study. Clin Microbiol Infect. 2010;16:1161-4.

26. Butler WR, O'Connor SP, Yakrus MA, Smithwick RW, Plikaytis BB, Moss CW, Floyd MM, Woodley CL, Kilburn JO, Vadney FS, et al. Mycobacterium celatum sp. nov. Int J Syst Bacteriol. 1993;43:539-48.

27. Bux-Gewehr I, Hagen HP, Rusch-Gerdes S, Feurle GE. Fatal pulmonary infection with Mycobacterium celatum in an apparently immunocompetent patient. J Clin Microbiol. 1998;36:587-8.

28. Miwa S, Shirai M, Toyoshima M, Shirai T, Yasuda K, Yokomura K, Yamada T, Masuda M, Inui N, Chida K. Efficacy of clarithromycin and ethambutol for Mycobacterium avium complex pulmonary disease. A preliminary study. Ann Am Thorac Soc. 2014;11:23-9.

29. Cespedes MS, Aberg JA. Mycobacterium avium Complex (MAC). Available from: http://www.antimicrobe.org/ms02.asp. Accessed 5 Dec 2015.

30. Zhao X, Wang Y, Pang Y. Antimicrobial susceptibility and molecular characterization of Mycobacterium intracellulare in China. Infect Genet Evol. 2014;27:332-8

\section{Submit your next manuscript to BioMed Central and we will help you at every step:}

- We accept pre-submission inquiries

- Our selector tool helps you to find the most relevant journal

- We provide round the clock customer support

- Convenient online submission

- Thorough peer review

- Inclusion in PubMed and all major indexing services

- Maximum visibility for your research

Submit your manuscript at www.biomedcentral.com/submit 